\title{
Duality in Josephson Junction Arrays
}

\author{
Ya. M. Blanter ${ }^{\mathrm{a}}$, Rosario Fazio ${ }^{\mathrm{b}}$, and Gerd Schön ${ }^{\mathrm{c}}$ \\ aDépartement de Physique Théorique, Université de Genève, CH-1211 Genève 4, Switzerland \\ bIstituto di Fisica, Facolta di Ingegneria, Università di Catania, 95128 Catania, Italy \\ c Institut für Theoretische Festkörperphysik, Universität Karlsruhe, 76128 Karlsruhe, Germany
}

Various properties of mesoscopic two-dimensional Josephson junction arrays are reviewed. Particular attention is paid to structure of the topological excitations, charges and vortices, which are shown to be dual to each other. This duality persists in the presence of external magnetic fields and offset charges, which influence vortices and charges in an equivalent way. A double-layer junction array is also considered, where an even further reaching duality is discovered.

\section{Introduction}

Since the original work by Kramers and Wannier [1] on the two-dimensional (2D) Ising model, duality has been proven to be a powerful tool in field theory and statistical mechanics [2]. The idea behind this transformation is the mapping of the weak coupling region of the system under consideration onto the strong coupling range (and vice versa). The symmetries of the system under this transformation lead to important insight into the structure of the model, especially in the intermediate region of coupling constants which is usually elusive to standard treatments. Dual transformations applied to the topological excitations of the system are particularly advantageous since it is possible to recast the partition function solely in terms of these degrees of freedom. José et al. [3] applied these techniques to show that the vortices in 2D XY-model can be mapped onto the charges of a two-dimensional Coulomb gas. Kadanoff [4] showed that this mapping is not restricted to the $\mathrm{XY}$-model, but various systems can be mapped onto coupled Coulomb gases. A review of these techniques applied to a number of systems can be found in Ref. [5].

In the past years two-dimensional Josephson junction arrays (JJA) have proven to be an excellent arena for the study of a variety of phase transitions [6]. They are fabricated from an array of superconducting islands connected by Joseph- son links. Each island is characterized by the modulus and the phase of the order parameter, $\Delta \exp (i \phi)$. Upon lowering the temperature, each island of the array goes superconducting at the BCS critical temperature $T_{\text {co }}$. But, in spite of the fact that each island is superconducting, the whole array remains in a resistive state as long as the phases, driven both by thermal and quantum fluctuations, have not acquired (sufficient) long range order. This global phase coherence sets in at a lower temperature. It is reasonable to assume that at these temperatures the magnitude of the order parameter is fixed to its equilibrium value, and the array can be described only in terms of the phases $\phi_{i}$. The resulting Hamiltonian is

$H=-E_{\mathbf{J}} \sum_{\langle i j\rangle} \cos \left(\phi_{i}-\phi_{j}\right)$

where $E_{\mathbf{J}}>0$ is the Josephson coupling energy, and the summation is over nearest neighbors. The lattice model defined in Eq.(1) is equivalent to the 2D XY-model - a planar lattice of localized planar magnetic momenta $\mathbf{S}_{\mathbf{i}}$ of unit length. It describes other physical systems as well, including planar ferromagnets, two-dimensional crystals [7] or two-dimensional superconducting films [8].

The XY-model undergoes a BerezinskiiKosterlitz-Thouless (BKT) transition [9,7,10] driven by fluctuations in the vortex density. For the JJA this implies that the array is phase coherent (superconducting) below the temperature $T_{\mathbf{J}}$, 
which is of the order of $E_{\mathbf{J}}$ (we use units where $\left.\hbar=k_{\mathrm{B}}=1\right)$. This is demonstrated by a simple argument due to Kosterlitz and Thouless. At zero temperature the lowest energy state of the system has the phases $\phi_{i}$ aligned, however, at finite temperatures the free energy $F=E-T S$ should be minimized. Consider an isolated vortex configuration. Its energy is readily calculated from Eq. (1). Logarithmic divergences are cut off at short distances by the lattice spacing $a$ and at long distances by the system size $R$, hence $E=\pi E_{\mathbf{J}} \ln (R / a)$. The entropy of the vortex configuration is given by the logarithm of the number of possible positions of the vortex, $S=2 \ln (R / a)$. Thus, the free energy of the vortex becomes negative at the temperature $T_{\mathbf{J}}=\pi E_{\mathbf{J}} / 2$. Below this temperature the phases are ordered, and vortices may appear only in bound pairs. I.e. the XYmodel is in a ferromagnetic state, while the JJA is superconducting. Above the transition temperature $T_{\mathbf{J}}$ free vortices may form. Their motion causes dissipation, and hence the state is resistive. This analysis is confirmed by the renormalization group calculations [11]. A thorough review concerning the statics and dynamics of the BKT transition can be found in Ref. [12].

\section{Charge-vortex duality in 2D Josephson junction arrays}

If the superconducting islands are of submicron size, as can be realized with modern nanolithography, the electrostatic charging energy associated with non-neutral configuration of the islands cannot be disregarded any longer. It can be expressed as

$E_{\mathrm{ch}}=\frac{1}{2} \sum_{i j} V_{i} C_{i j} V_{j}$.

Here the summation is over all islands $i$ with local voltage $V_{i}=\partial_{t}\left(\phi_{i} / 2 e\right)$. The capacitance matrix is determined in a good approximation by the on-site and the nearest neighbors elements, $C_{i i}=C_{0}+4 C, C_{i j}=-C$ if $i$ and $j$ are nearestneighbors, and $C_{i j}=0$ otherwise. Here $C_{0}$ is the capacitance of the grains to the ground while $C$ is the junction capacitance.
In a quantum mechanical treatment, the charge $\hat{Q}_{i}$ on the island $i$ has to be viewed as conjugated to the phase $\phi_{i}$ (see e.g. Ref. [13]), i.e.

$\hat{Q}_{i}=\frac{1}{i} \frac{\partial}{\partial\left(\phi_{i} / 2 e\right)}$.

Then the Hamiltonian of the array is [14]

$$
\hat{H}=\frac{1}{2} \sum_{i j} \hat{Q}_{i} C_{i j}^{-1} \hat{Q}_{j}-E_{\mathbf{J}} \sum_{\langle i j\rangle} \cos \left(\phi_{i}-\phi_{j}\right) .
$$

The interaction between the charges in the array is determined by the inverse capacitance matrix $C_{i j}^{-1}$. Its range depends on the ratio $C / C_{0}$; if the capacitance to ground is much larger than the junction capacitance the charges feel an on-site interaction. In the opposite limit the Coulomb interaction depends logarithmically on the distance. The capacitance to the ground $C_{0}$ leads to a screening on the length scale $\sim a \sqrt{C / C_{0}}$.

The two characteristic energy scales in the system are the Josephson coupling energy $E_{\mathbf{J}}$, which is associated to the tunneling of Cooper pairs between neighboring islands, and the charging energy $E_{\mathrm{C}}=e^{2} /\left(2 \max \left\{C_{0}, C\right\}\right)$, which is the energy cost to add an extra Cooper pair on a neutral island. The charging energy $E_{\mathrm{C}}$ tends to inhibit Josephson tunneling and is responsible for quantum fluctuations of the phases $\phi_{i}$ on each island. If $E_{\mathbf{J}} \gg E_{\mathrm{C}}$ the fluctuations of the phases are weak, and the system acquires global superconducting coherence.

In this section we derive some properties of quantum JJA from duality arguments. Our starting point is the partition function,

$$
\begin{aligned}
Z= & \prod_{i} \int_{0}^{2 \pi} d \phi_{i}^{(0)} \sum_{\left\{m_{i}=0, \pm 1, \ldots\right\}} \\
& \int_{\phi_{i}^{(0)}}^{\phi_{i}^{(0)}+2 \pi m_{i}} D \phi_{i}(\tau) \exp (-S\{\phi\}) .
\end{aligned}
$$

Here the Euclidean path integration is carried out with the boundary conditions

$\phi_{i}(0)=\phi_{i}^{(0)} ; \quad \phi_{i}(\beta)=\phi_{i}^{(0)}+2 \pi m_{i}$,

with $\beta$ being the inverse temperature. These nontrivial boundary conditions express the fact that 
the charges of the grains are integer multiples of $2 e[15,13]$. The Euclidean effective action $S\{\phi\}$ has the form

$$
\begin{aligned}
S\{\phi\}=\int_{0}^{\beta} d \tau\left\{\frac{C_{0}}{8 e^{2}} \sum_{i}\left(\dot{\phi}_{i}\right)^{2}+\frac{C_{1}}{8 e^{2}} \sum_{\langle i j\rangle}\left(\dot{\phi}_{i}-\dot{\phi}_{j}\right)^{2}\right. \\
\left.-E_{\mathbf{J}} \sum_{\langle i j\rangle} \cos \left(\phi_{i}-\phi_{j}\right)\right\}
\end{aligned}
$$

The model has been studied extensively to derive the phase diagram by means of mean field [16-20], Monte Carlo [21], variational [22] and RG [23] calculations. Non-perturbative features of this model and its connections to planar Chern-Simons gauge theories were discussed in Ref. [24]. Quantum fluctuations lower the temperature of the BKT phase transition separating resistive and superconductive phases [23] below the transition temperature $T_{\mathbf{J} 0}$ of the classical array (with $E_{\mathrm{C}}=0$ ). In the case $C_{0}=0$ the shift of the transition temperature is

$T_{\mathbf{J}}=T_{\mathbf{J} 0}-\frac{e^{2}}{48 \pi C}$.

Beyond a critical value of the charging energy $E_{\mathrm{C}}$ the transition temperature vanishes, and the array remains insulating even at zero temperature.

The purpose of this Chapter is to show that there is a dual transformation relating the classical limit, $E_{\mathbf{J}} \gg E_{\mathbf{C}}$, to the opposite quantum limit, $E_{\mathbf{J}} \ll E_{\mathrm{C}}[25,26,14,27]$. In the latter case the quantum fluctuations of the phases are strong, and vortices are ill-defined objects. However, in this regime the charges on the islands are welldefined variables. In the extreme limit $C_{0} \ll C$, the interaction between the charges is logarithmic, in the same way as that of the vortices in the classical array. In this case the charges form a 2D Coulomb gas and undergo a BKT transition at temperature $T_{\mathrm{C}} \sim E_{\mathrm{C}}$, where the state below the transition is insulating. If the capacitance to the ground is larger, no finite-temperature phase transition arises. However, we can still expect a crossover from a low-temperature phase with exponentially low conductance to a resistive phase at finite temperature.

In order to describe an intermediate situation we need a formulation in terms of both charges and vortices. Following Ref. [14,27] we first introduce the island charges in a path integral representation. In terms of phase trajectories $\phi_{i}(\tau)$ and charges $q_{i}(\tau)=Q_{i}(\tau) / 2 e$ the partition function takes the form

$$
\begin{array}{r}
Z=\prod_{j} \int d q_{j 0} \int D q_{j}(\tau) \prod_{i} \int_{0}^{2 \pi} d \phi_{i}^{(0)} \sum_{\left\{m_{i}\right\}} \\
\int D \phi_{i}(\tau) \exp (-S\{\phi, q\}),
\end{array}
$$

where the phases obey the boundary conditions (6), while the charge paths are periodic, $q_{j}(0)=$ $q_{j}(\beta)=q_{j 0}$. The effective action in terms of phases $\phi_{i}(\tau)$ and charges $q_{i}(\tau)$ is

$$
\begin{aligned}
S\{\phi, q\} & =\int_{0}^{\beta} d \tau\left\{2 e^{2} \sum_{i, j} q_{i} C_{i j}^{-1} q_{j}\right. \\
& \left.+i \sum_{i} q_{i} \dot{\phi}_{i}-E_{\mathbf{J}} \sum_{\langle i j\rangle} \cos \left(\phi_{i}-\phi_{j}\right)\right\}
\end{aligned}
$$

The summation over winding numbers $\left\{m_{i}\right\}$ fixes the charges $q_{i}$ to be integer-valued [15].

Following the steps discussed in the Appendix, the partition function can be expressed as a sum over charge and vortex configurations

$$
Z=\sum_{\left\{q_{i \tau}\right\}} \sum_{\left\{v_{i \tau}\right\}} \exp (-S\{q, v\})
$$

The effective action for the integer charges $q_{i}(\tau)$ and vorticities $v_{i}(\tau)$ is

$$
\begin{aligned}
S\{q, v\} & =\int_{0}^{\beta} d \tau \sum_{i j}\left\{2 e^{2} q_{i} C_{i j}^{-1} q_{j}+\pi E_{\mathbf{J}} v_{i} G_{i j} v_{j}\right. \\
& \left.+i q_{i} \Theta_{i j} \dot{v}_{j}+\frac{1}{4 \pi E_{\mathbf{J}}} \dot{q}_{i} G_{i j} \dot{q}_{j}\right\} .
\end{aligned}
$$

It describes two coupled Coulomb gases. The charges interact via the inverse capacitance matrix. The interaction among the vortices is described by the kernel $G_{i j}$, which is obtained as the Fourier transform of $k^{-2}$. At large distances $r_{i j} \gg a$ between the sites $i$ and $j$ it depends logarithmically on the distance

$G_{i j}=\frac{1}{2} \ln \left(\frac{a}{r_{i j}}\right)$. 
The charges and vortices are coupled in the dynamical theory by the third term. Here

$\Theta_{i j}=\arctan \left(\frac{y_{i}-y_{j}}{x_{i}-x_{j}}\right)$

describes the phase configuration at the site $i$ if a vortex is placed at the site $j$. The coupling has a simple physical interpretation: a change of vorticity at site $j$ produces a voltage at site $i$ which is felt by the charge at this location. The last term $\dot{q} G \dot{q}$ represents a spin-wave contribution to the charge correlation function.

The effective action (12) shows a high degree of symmetry between vortex and charge degrees of freedom. In particular, in the limit $C_{0} \ll C$ the inverse capacitance matrix depends on distance in the same way as the vortex interaction,

$e^{2} C_{i j}^{-1}=\frac{E_{\mathrm{C}}}{\pi} G_{i j}$,

and charges and vortices are (nearly) dual. The duality is broken by the last term $\dot{q} G \dot{q}$. This term is "irrelevant" for the phase transitions, i.e. it merely shifts the transition point. But it has important consequences for the dynamical behavior.

The action (12) is written in terms of integer charges and vorticities. However, depending on the coupling constants, only one type of excitations may be well defined. In the quasiclassical limit, $E_{\mathbf{J}} \gg E_{\mathrm{C}}$, vortices are the relevant excitations, while charges are strongly fluctuating and can be treated as continuous variables. By integrating out the charges, one obtains the effective action for vortices $v_{i}(\tau)$

$$
\begin{array}{r}
S\{v\}=\int_{0}^{\beta} d \tau\left\{\frac{1}{8 e^{2}} \sum_{i j k l} \dot{v}_{i} \Theta_{i k} C_{k l} \Theta_{l j} \dot{v}_{j}\right. \\
\left.+\pi E_{\mathbf{J}} \sum_{i j} v_{i} G_{i j} v_{j}\right\} .
\end{array}
$$

In the limit of low self-capacitances, $C_{0} \ll C$, the kernel in the first term becomes $\Theta_{i k} G_{k l}^{-1} \Theta_{l j}$, and the effective action for vortices reduces to [28]

$$
S\{v\}=\int_{0}^{\beta} d \tau \sum_{i j}\left\{\frac{\pi}{8 E_{\mathrm{C}}} \dot{v}_{i} G_{i j} \dot{v}_{j}\right.
$$

$$
\left.+\pi E_{\mathbf{J}} \sum_{i j} v_{i} G_{i j} v_{j}\right\} .
$$

The summation in the partition function is constrained by the neutrality condition $\sum_{i} v_{i}=0$.

The effective action (17) describes a quantum Coulomb gas of vortices. This becomes clearer if we change from a description in terms of the vorticity at site $i$ to a continuous description where we label the vortices by their center coordinate $\mathbf{r}(\tau)$ and sign of vorticity $v_{n}= \pm 1$ [29]. Both are related by

$v_{i}(\tau) \rightarrow \sum_{n} v_{n} \delta\left(\mathbf{r}_{i}-\mathbf{r}_{n}(\tau)\right)$.

In these variable the partition function becomes,

$Z=\sum_{N=0}^{\infty} \int D \mathbf{r}_{1}(\tau) \ldots \mathbf{r}_{2 N}(\tau) \exp (-S\{\mathbf{r}\})$.

(We have explicitly used the charge neutrality conditions: the integration is carried over $N$ vortices with $v=1$ and $N$ anti-vortices with $v=$ $-1)$. The effective action expressed in terms of the vortex coordinates is

$$
\begin{aligned}
S\{\mathbf{r}\}=\int_{0}^{\infty} d \tau \sum_{m, n=1}^{2 N} & {\left[\frac{1}{2} \dot{r}_{m}^{\alpha} M_{\alpha \beta}\left(\mathbf{r}_{m}-\mathbf{r}_{n}\right) \dot{r}_{n}^{\beta}\right.} \\
& \left.+\pi E_{\mathbf{J}} G\left(\mathbf{r}_{m}-\mathbf{r}_{n}\right)\right] .
\end{aligned}
$$

The second term in (20) is the interaction energy of the vortices. In the first term we introduced the vortex mass tensor [30],

$M_{\alpha \beta}(\mathbf{r})=-\frac{\pi}{4 E_{\mathrm{C}}} \nabla_{\alpha} \nabla_{\beta} G(\mathbf{r})$.

For $r \gg a$ it decreases as $r^{-2}$, and consequently may be approximated by a local function

$M_{\alpha \beta}(\mathbf{r})=M_{\mathrm{v}} \delta_{\alpha \beta} \delta(\mathbf{r}), \quad M_{\mathrm{v}}=\frac{\pi^{2}}{4 a^{2} E_{\mathrm{C}}}$.

It defines the vortex mass $M_{\mathrm{v}}[30,31]$. In this case the first term represents a kinetic energy of vortices, which can be rewritten as

$S_{\mathrm{kin}}^{(\mathrm{v})}=\int_{0}^{\beta} d \tau \sum_{m=1}^{2 N} \frac{1}{2} M_{\mathrm{v}} \dot{r}_{m}^{2}(\tau)$. 
A similar consideration for $E_{\mathrm{C}} \gg E_{\mathbf{J}}$ lead us to the action of a 2D Coulomb gas of charges with charge mass $M_{\mathrm{q}}=\left(a^{2} E_{\mathbf{J}}\right)^{-1}$ (see also Ref. [32]). Thus, in two dimensional arrays of Josephson junctions a charge-vortex duality exists. In the limit $E_{\mathbf{J}} \gg E_{\mathrm{C}}$ the vortices are well-defined. They form a Coulomb gas, and can be considered as particles with masses. In the opposite limit $E_{\mathrm{C}} \ll E_{\mathbf{J}}$ the charges are the relevant excitations. The charges have the same properties as the vortices in the corresponding limiting cases.

\section{Double-layered arrays}

Another system of increasing experimental interest is composed of two parallel 2D Josephson junction arrays with purely capacitive coupling between them (no Josephson coupling [33]). We will show that in this case an even further reaching duality between charges and vortices arises. We restrict ourselves to the most interesting situation, when one array is in the quasi-classical (vortex) regime while the other is in the quantum (charge) regime. Then the vortices in one layer and the charges in the other are simultaneously well-defined dynamical variables. (In contrast in a single array vortices or charges acquire a kinetic energy only after the other variable is integrated out.) Another important feature of the present system is that the strength of interaction between charges and vortices is controlled by the interlayer coupling $C_{\mathrm{x}}$ and consequently may be tuned independently. We show that the physical realization of this interaction is rather different from that in a single array. The theoretical description of this system has been developed in Refs. [34,35].

The partition function of the system in terms of the phases $\phi_{i \mu}$ (the indices $i$ label the islands in each array and $\mu=1,2$ refers to the layer) is

$$
\begin{aligned}
& Z=\prod_{i} \int_{0}^{2 \pi} d \phi_{i 1}^{(0)} d \phi_{i 2}^{(0)} \sum_{\left\{m_{i 1}\right\},\left\{m_{i 2}\right\}} \\
& \int D \phi_{i 1}(\tau) D \phi_{i 2}(\tau) \exp \left(-S\left\{\phi_{1}, \phi_{2}\right\}\right),
\end{aligned}
$$

with boundary conditions $\phi_{i \mu}(0)=\phi_{i \mu}^{(0)}$ and $\phi_{i \mu}(\beta)=\phi_{i \mu}^{(0)}+2 \pi m_{i \mu}$. The Euclidean effective action $S\left\{\phi_{1}, \phi_{2}\right\}$ has the form

$$
\begin{gathered}
S\left\{\phi_{1}, \phi_{2}\right\}=\int_{0}^{\beta} d \tau\left\{\sum _ { \mu = 1 , 2 } \left[\frac{C_{0 \mu}}{8 e^{2}} \sum_{i}\left(\dot{\phi}_{i \mu}\right)^{2}\right.\right. \\
\left.+\frac{C_{\mu}}{8 e^{2}} \sum_{\langle i j\rangle}\left(\dot{\phi}_{i \mu}-\dot{\phi}_{j \mu}\right)^{2}-E_{\mathbf{J}} \sum_{\langle i j\rangle} \cos \left(\phi_{i \mu}-\phi_{j \mu}\right)\right] \\
\left.+\frac{C_{\mathrm{x}}}{8 e^{2}} \sum_{i}\left(\dot{\phi}_{i 1}-\dot{\phi}_{i 2}\right)^{2}\right\} .
\end{gathered}
$$

Here $C_{0 \mu}$ are the capacitances of the islands in the array $\mu$ relative to the ground, $C_{\mu}$ are the capacitances of the junctions in the array $\mu$, and $C_{\mathrm{x}}$ are the interlayer capacitances between adjacent islands, while $E_{\mathbf{J}_{\mu}}$ are the Josephson coupling constants in the layers.

We concentrate on the situation in which the array 1 is in the quantum (charge) regime while the array 2 is in the quasi-classical (vortex) regime, i.e.

$$
E_{\mathbf{J} 1} \ll e^{2} / 2 \tilde{C}_{1}, \quad E_{\mathbf{J} 2} \gg e^{2} / 2 \tilde{C}_{2},
$$

with $\tilde{C}_{\mu}=\max \left\{C_{0 \mu}, C_{\mu}, C_{\mathrm{x}}\right\}$. Various types of problems van be studied in these systems. If we are interested only in behavior of the vortex array (e.g. in an experimental realization where one measures the quantities in the vortex array only) we can integrate out all degrees of freedom related to the charge array and study an effective action for the vortex array. An analysis analogous to that presented for a single array shows that this action is essentially that of the Coulomb gas [34]. Consequently the vortex array undergoes a BKT transition, and in the quasiclassical regime its temperature is lowered due to electrostatic coupling to the charge array.

A similar problem can be solved for the charge array. Then the charge-BKT transition changes into a crossover, since the capacitive coupling to the vortex array leads to screening similar as capacitances to the ground.

Our purpose here is to consider both arrays simultaneously and to investigate the charge-vortex duality in this system. Similar as in a single array, we move from a description in terms of phases to one in terms of charges and vortices, and 
use the duality of the resulting action to investigate the transition. We will show that charges and vortices in this system can be considered as two-dimensional dynamical particles with masses. The charge-charge and vortex-vortex interaction are essentially those of 2D Coulomb particles, while the charge-vortex interaction is highly interesting.

Before we proceed with the calculation it is necessary to stress the following. In the regime of interest the interlayer capacitances $C_{\mathrm{x}}$ not only couple the layers, but also renormalize the capacitances $C_{01}$ and $C_{02}$ of the islands to the ground. The physical reason for this is that due to the strong fluctuations of charges in layer 2 and vorticities in layer 1 these variables are effectively continuous, and hence a coupling to the other array plays the same role as a coupling to the ground. Hence the interaction between the charges in each layer has a finite range for any non-zero $C_{\mathrm{x}}$ due to the screening, and the BKT transition is replaced by a crossover. On the other hand, in the limit $C_{01} \ll C_{\mathrm{x}} \ll C_{1}$ the screening length $\xi_{1} \sim a\left(C_{1} / C_{\mathrm{X}}\right)^{1 / 2}$ can be very large. Below we assume that these inequalities are satisfied and the range of interaction $\xi_{1}$ is large enough to make it meaningful to speak about the chargeunbinding transition. This just means that the crossover is (exponentially) sharp. For not so weak coupling $C_{\mathrm{x}}$ this description becomes meaningless, since the crossover is strongly smeared, and the insulating phase is absent.

To proceed it is convenient to introduce the large capacitance matrix

$\hat{C}=\left(\begin{array}{cc}\hat{C}_{1} & -\hat{C}_{\mathrm{x}} \\ -\hat{C}_{\mathrm{x}} & \hat{C}_{2}\end{array}\right)$.

Here $\hat{C}_{\mu}$ is the capacitance matrix in the array $\mu$ while $\left(\hat{C}_{\mathrm{x}}\right)_{i j}=C_{\mathrm{x}} \delta_{i j}$. The inverse matrix, describing the interaction of charges, in the Fourier representation reads as

$$
\begin{aligned}
& \hat{C}^{-1}(\mathrm{k})=\left\{\prod_{\mu=1,2}\left(C_{0 \mu}+C_{\mu} \mathrm{k}^{2}+C_{\mathrm{x}}\right)-C_{\mathrm{x}}^{2}\right\}^{-1} \\
& \times\left(\begin{array}{cc}
C_{02}+C_{2} \mathrm{k}^{2}+C_{\mathrm{x}} & C_{\mathrm{x}} \\
C_{\mathrm{x}} & C_{01}+C_{1} \mathrm{k}^{2}+C_{\mathrm{x}}
\end{array}\right) \cdot(27)
\end{aligned}
$$

The effective action (25) can be rewritten in terms of integer charges $q_{i \mu}(\tau)$ and phases $\phi_{i \mu}(\tau)$ of each island

$$
\begin{aligned}
& S\{q, \phi\}=\int_{0}^{\beta} d \tau\left\{2 e^{2} \sum_{i j, \mu, \nu} q_{i \mu}\left(\hat{C}^{-1}\right)_{i j}^{\mu \nu} q_{j \nu}\right. \\
& \left.+i \sum_{i \mu}\left[q_{i \mu} \dot{\phi}_{i \mu}-E_{\mathbf{J}} \sum_{\langle i j\rangle} \cos \left(\phi_{i \mu}-\phi_{j \mu}\right)\right]\right\} .
\end{aligned}
$$

It is possible to introduce vortex degrees of freedom in the same way as for one array. Since this procedure deals only with the phase variables and does not affect the charge interaction (the first term in Eq. (28)), the generalization for doublelayered arrays is trivial. We obtain

$$
Z=\sum_{\left\{q_{i 1}(\tau), q_{i 2}(\tau)\right\}} \sum_{\left\{v_{i 1}(\tau), v_{i 2}(\tau)\right\}} \exp (-S\{q, v\}),
$$

where the effective action for integer charges $q_{i \mu}$ and vorticities $v_{i \mu}$ is

$$
\begin{array}{r}
S\{q, v\}=\int_{0}^{\beta} d \tau\left\{2 e^{2} \sum_{i j, \mu, \nu} q_{i \mu}\left(\hat{C}^{-1}\right)_{i j}^{\mu \nu} q_{j \nu}\right. \\
+\sum_{\mu}\left[\frac{1}{4 \pi E_{\mathbf{J} \mu} F\left(\epsilon_{\mu} E_{\mathbf{J} \mu}\right)} \sum_{i j} \dot{q}_{i \mu} G_{i j} \dot{q}_{j \mu}\right. \\
+\pi E_{\mathbf{J} \mu} F\left(\epsilon_{\mu} E_{\mathbf{J} \mu}\right) \sum_{i j} v_{i \mu} G_{i j} v_{j \mu} \\
\left.\left.+i \sum_{i j} \dot{q}_{i \mu} \Theta_{i j} v_{j \mu}\right]\right\} .
\end{array}
$$

Here we wrote terms which arise from the discretization of the time with lattice spacing (in array $\mu$ ) of order $\epsilon_{\mu} \sim\left(8 E_{\mathbf{J} \mu} E_{\mathrm{C} \mu}\right)^{-1 / 2}$ and $E_{\mathrm{C} \mu} \equiv$ $e^{2} / 2 C_{\mu}$. (See the Appendix for the function $F$ renormalizing the Josephson coupling). In the limit considered only the Josephson coupling of array 1 is noticeably renormalized,

$\tilde{E}_{\mathbf{J} 1} \sim\left(8 E_{\mathbf{J} 1} E_{\mathrm{C} 1}\right)^{1 / 2}\left[\ln \left(E_{\mathrm{C} 1} / E_{\mathbf{J} 1}\right)\right]^{-1}$.

We suppress the tilde from now on. Later we will assume that the linear size of each array is much less than the range of interaction $\xi_{\mu}=$ $a\left(C_{\mu} / C_{\mathrm{x}}\right)^{1 / 2}$. This means, in particular, that we assume $C_{\mathrm{x}} \ll C_{2}$. 
The action (30) depends on the charges and vorticities in both layers. However, in our situation, when the layers 1 and 2 are in the charge and vortex regimes, respectively, the vortices in the layer 1 and the charges in the layer 2 are strongly fluctuating degrees of freedom and may be integrated out. To do this we suppose the latter variables to be continuous and neglect the spin-wave charge coupling term, $\dot{q}_{2} G \dot{q}_{2}$, in layer 2. Then after performing the Gaussian integration we obtain the effective action for charges $q_{i 1}(\tau)$ in layer 1 and vorticities $v_{i 2}(\tau)$ in layer 2 (to be referred below as $q_{i}$ and $v_{i}$ )

$$
\begin{aligned}
& S\{q, v\}=\int_{0}^{\beta} d \tau\left\{2 e^{2} \sum_{i j} q_{i}\left(\hat{C}_{i j}^{-1}\right)_{i j}^{11} q_{j}\right. \\
&+ \frac{1}{4 \pi E_{\mathbf{J} 1}} \sum_{i j} \dot{q}_{i} G_{i j} \dot{q}_{j}+\pi E_{\mathbf{J} 2} \sum_{i j} v_{i} G_{i j} v_{j} \\
&+\frac{1}{8 E e^{2}} \sum_{i j k l} \dot{v}_{i} \Theta_{i k}\left[\left(\hat{C}^{-1}\right)^{22}\right]_{k l}^{-1} \Theta_{l j} \dot{v}_{j} \\
&\left.+i \sum_{i j k l} \dot{v}_{i} \Theta_{i k}\left[\left(\hat{C}^{-1}\right)^{22}\right]_{k l}^{-1}\left[\left(\hat{C}^{-1}\right)^{12}\right]_{l j}^{-1} q_{j}\right\} .
\end{aligned}
$$

In the limit where the charge interaction is longrange we can use the appropriate limits of the large capacitance matrix (27). In this case the action reduces to

$$
\begin{gathered}
S\{q, v\}=\int_{0}^{\beta} d \tau\left\{\frac{2 E_{\mathrm{C} 1}}{\pi} \sum_{i j} q_{i} G_{i j} q_{j}\right. \\
+\frac{1}{4 \pi E_{\mathbf{J} 1}} \sum_{i j} \dot{q}_{i} G_{i j} \dot{q}_{j}+\pi E_{\mathbf{J} 2} \sum_{i j} v_{i} G_{i j} v_{j} \\
+\frac{\pi}{8 E_{\mathrm{C} 2}} \sum_{i j} \dot{v}_{i}\left[G_{i j}-\frac{C_{\mathrm{x}}^{2}}{4 \pi^{2} C_{1} C_{2}} \sum_{k l} \Theta_{i k} G_{k l} \Theta_{l j}\right] \dot{v}_{j} \\
\left.+\frac{i C_{\mathrm{x}}}{2 \pi C_{1}} \sum_{i j k} \dot{v}_{i} \Theta_{i k} G_{k j} q_{j}\right\} .
\end{gathered}
$$

This form displays the duality between charges and vortices in the appropriate limit.

The action (33) is the central result of this section. It looks rather similar to the effective charge-vortex action in one Josephson junction array. The most important difference is that in one layer either charges or vortices are welldefined degrees of freedom, while the action (33) of the double-layer array describes the system of two well-defined dynamic variables on each site the charges in layer 1 and the vortices in layer 2 . The action shows a duality between charges and vortices (the second term in the square brackets is small for $\left.C_{\mathrm{x}} \ll C_{1}, C_{2}\right)$. Both kinetic terms for charges and vortices violate the duality due to the numerical coefficients. However, close enough to the transitions these terms produce only small renormalization of the transition temperature, and are irrelevant. Another interesting feature of this action is that the last term, describing the interaction between charges and vortices, is also small, while in a single-layer array the interaction is always of the same order of magnitude as the other terms.

It is obvious that for long-range interaction of the charges in layer 1 these also exhibit a BKT transition, and under the conditions where the action (33) was obtained the transition temperature does not feel the presence of layer 2. Hence

$$
T_{\mathrm{C}}=T_{\mathrm{C} 0}-\frac{E_{\mathbf{J} 1}}{24 \pi} .
$$

To understand the physics described by the action (33) it is instructive to map this model onto the 2D Coulomb gas. For this purpose we move again from the space-time lattice to the continuous medium and introduce the coordinates of the vortex centers and charges

$$
\begin{aligned}
& q_{i}(\tau) \rightarrow \sum_{m} q_{m} \delta\left(\mathbf{r}-\mathbf{r}_{m}(\tau)\right) \\
& v_{i}(\tau) \rightarrow \sum_{n} v_{n} \delta\left(\mathbf{r}-\mathbf{R}_{n}(\tau)\right) .
\end{aligned}
$$

Here $q_{m}= \pm 1$ and $v_{n}= \pm 1$ represent charges and vortices, respectively, and $\mathbf{r}_{m}(\tau)$ and $\mathbf{R}_{n}(\tau)$ the coordinates of their centers. In terms of these variables the partition function reads

$$
\begin{aligned}
Z=\sum_{M=0}^{\infty} \sum_{N=0}^{\infty} \int D \mathbf{r}_{1}(\tau) \ldots D \mathbf{r}_{2 M}(\tau) \\
D \mathbf{R}_{1}(\tau) \ldots D \mathbf{R}_{2 N}(\tau) \exp (-S\{\mathbf{r}, \mathbf{R}\}),
\end{aligned}
$$


with an effective action $S\{\mathbf{r}, \mathbf{R}\}$ describing a neutral system of $2 M$ positive and negative charges $(q= \pm 1)$ and of $2 N$ positive and negative vortices $(v= \pm 1)$.

The first and third terms of the action (33) can be easily transformed by means of decomposition (34). The first one produces the potential energy of charge interaction,

$S_{\text {int }}^{(q)}=$

$\frac{2 E_{\mathrm{C} 1}}{\pi} \int_{0}^{\beta} d \tau \sum_{m, n=1}^{2 M} q_{m} q_{n} G\left(\mathbf{r}_{m}(\tau)-\mathbf{r}_{n}(\tau)\right)$.

In principle, the summation includes the terms with $m=n$; these, however, give rise only to the chemical potential for charges. The third term in Eq. (33) yields the interaction of vortices,

$S_{\text {ivt }}^{(\mathrm{v})}=$

$\pi E_{\mathbf{J} 2} \int_{0}^{\beta} d \tau \sum_{m, n=1}^{2 N} v_{m} v_{n} G\left(\mathbf{R}_{m}(\tau)-\mathbf{R}_{n}(\tau)\right)$.

Here again the term with $m=n$ gives rise to the chemical potential for vortices. The terms (36) and (37) are essentially the action for (classical) Coulomb gases of charges and vortices, respectively [11].

If we neglect the small correction proportional to the $C_{\mathrm{x}}^{2} / C_{1} C_{2}$ in the fourth term in Eq.(33) then the second and fourth terms can be transformed to the kinetic energy of charges and vortices respectively [14]. The second term gives

$S_{\text {kin }}^{(\mathrm{q})}=\frac{1}{2} \int_{0}^{\beta} d \tau \sum_{m, n=1}^{2 M} q_{m} q_{n} \dot{r}_{m}^{\gamma} M_{\gamma \delta}\left(\mathbf{r}_{m}-\mathbf{r}_{n}\right) \dot{r}_{n}^{\delta}$,

where the charge mass tensor can be approximated as

$$
\begin{aligned}
M_{\gamma \delta}(\mathbf{r}) & =-\frac{1}{\pi E_{\mathbf{J} 1}} \nabla_{\gamma} \nabla_{\delta} G(\mathbf{r}) \approx M_{\mathrm{q}} \delta_{\gamma \delta} \delta(\mathbf{r}) \\
M_{\mathrm{q}} & =\frac{1}{a^{2} E_{\mathbf{J} 1}} .
\end{aligned}
$$

Then the kinetic term for charges takes a simple form

$S_{\mathrm{kin}}^{(\mathrm{q})}=\int_{0}^{\beta} d \tau \sum_{m=1}^{2 M} \frac{1}{2} M_{\mathrm{q}} \dot{r}_{m}^{2}(\tau)$.
Similarly, the fourth term in Eq.(33) produces the kinetic term for vortices

$$
\begin{array}{ll}
S_{\text {kin }}^{(\mathrm{v})}= & \int_{0}^{\beta} d \tau \sum_{m=1}^{2 N} \frac{1}{2} M_{\mathrm{v}} \dot{R}_{m}^{2}(\tau) \\
\text { with } & M_{\mathrm{v}}=\frac{\pi^{2}}{4 a^{2} E_{\mathrm{C} 2}} .
\end{array}
$$

Finally, the last term in Eq.(33)) is responsible for the interaction between charges and vortices. The corresponding term in the action is

$$
\begin{aligned}
& S_{\mathrm{qv}}=\frac{i C_{\mathrm{x}}}{2 \pi C_{1} a^{2}} \int_{0}^{\beta} d \tau \sum_{m n} v_{m} q_{n} \\
& \quad \int d \mathbf{r}^{\prime} \nabla_{\mathbf{R}_{m}} \Theta\left(\mathbf{R}_{m}-\mathbf{r}^{\prime}\right) G\left(\mathbf{r}^{\prime}-\mathbf{r}_{n}\right) \dot{\mathbf{R}}_{m}(\tau) .
\end{aligned}
$$

After the integration over $\mathbf{r}^{\prime}$ it reduces to

$S_{\mathrm{qv}}=-\int_{0}^{\beta} d \tau \sum_{m} i v_{m} \dot{\mathbf{R}}_{m}(\tau) \mathbf{A}\left(\mathbf{R}_{m}\right)$

with

$$
\mathbf{A}\left(\mathbf{R}_{m}\right)=\sum_{n} q_{n} \mathbf{a}\left(\mathbf{R}_{m}(\tau)-\mathbf{r}_{n}(\tau)\right),
$$

and

$\mathbf{a}(\mathbf{r})=-\frac{1}{8 a^{2}} \frac{C_{\mathrm{x}}}{C_{1}}\left[1+2 \ln \left(\frac{a}{r}\right)\right][\hat{z} \times \mathbf{r}]$.

The charges are the sources of a vector potential felt by the moving vortices. It depends of the signs of the corresponding vortices and charges. We can rewrite this term, after a partial integration in Eq.(33), in such a form that the vortices create a gauge potential for the charges. Hence this charge-vortex interaction term fully preserves the duality between charges and vortices.

In summary the resulting action

$$
S\{\mathbf{r}, \mathbf{R}\}=S_{\mathrm{int}}^{(\mathrm{q})}+S_{\mathrm{int}}^{(\mathrm{v})}+S_{\mathrm{kin}}^{(\mathrm{q})}+S_{\mathrm{kin}}^{(\mathrm{v})}+S_{\mathrm{qv}}
$$

is essentially that of two coupled 2D Coulomb gases. It is symmetric with respect to charges and vortices. Both can be considered as particles with masses

$M_{\mathrm{q}}=\frac{1}{a^{2} E_{\mathbf{J} 1}}$ and $M_{\mathrm{v}}=\frac{\pi^{2}}{4 a^{2} E_{\mathrm{C} 2}}$ 
respectively. Charges interact via the effective capacitance, vortices via the usual logarithmic interaction with strength $E_{\mathbf{J} 2}$. In addition, there is a specific feature of two-layered system: the vortices produce a vector potential a for the charges, whereas charges create a gauge potential for vortices. The magnetic field, associated with this vector potential is

$B= \pm \frac{1}{2 e a^{2}} \frac{C_{\mathrm{x}}}{C_{1}} \ln \frac{a}{r}, \quad a \ll r \ll \xi_{1}$.

The interaction described by this vector/gauge potential is always controlled by the small parameter $C_{\mathrm{x}} / C_{1}$. In the regime considered it is weak.

\section{Magnetic field - offset charge duality}

In this last section we return to the case of a single Josephson junction array. We assume that the array is in a perpendicular position-dependent magnetic field $\mathbf{B}_{i}$. Furthermore, we allow for "offset" charges on the grains $Q_{x i}$, which in principle can be controlled by applied gate voltages between the ground and the islands. (In practice they are caused e.g. by random impurity charges in the substrate.) Both these factors can be accounted in the formalism described above. The action describing this system is (see e.g. [36])

$$
\begin{aligned}
& S\{\phi, q\}= \\
& \int_{0}^{\beta} d \tau\left\{2 e^{2} \sum_{i j}\left[q_{i}(\tau)+q_{\times i}\right] C_{i j}^{-1}\left[q_{j}(\tau)+q_{x j}\right]\right. \\
& \left.+i \sum_{i} q_{i}(\tau) \dot{\phi}_{i}(\tau)-E_{\mathbf{J}} \sum_{\langle i j\rangle} \cos \left[\phi_{i}-\phi_{j}-A_{i j}\right]\right\} .
\end{aligned}
$$

Here $q_{\mathrm{x} i}=Q_{\mathrm{x} i} / 2 e$, and the magnetic field is taken into account by the vector-potential $A_{i j}=$ $(2 e / c) \int_{i}^{j} \mathbf{A} \cdot \mathbf{d l}$. After the same transformations as those performed in Sec. 2, we arrive to the coupled-Coulomb gas action for charges and vorticities, similar to Eq. (12)

$$
\begin{aligned}
& S\{q, v\}= \\
& \int_{0}^{\beta} d \tau \sum_{i j}\left\{2 e^{2}\left[q_{i}(\tau)+q_{\mathrm{x} i}\right] C_{i j}^{-1}\left[q_{j}(\tau)+q_{\mathrm{x} j}\right] .\right.
\end{aligned}
$$

$$
\begin{gathered}
+\pi E_{\mathbf{J}}\left[v_{i}(\tau)+f_{i}\right] G_{i j}\left[v_{j}(\tau)+f_{j}\right] \\
\left.+i\left[q_{i}(\tau)+q_{\mathrm{x} i}\right] \Theta_{i j} \dot{ }_{j}(\tau)+\frac{1}{4 \pi E_{\mathbf{J}}} \dot{q}_{i}(\tau) G_{i j} \dot{q}_{j}(\tau)\right\} .
\end{gathered}
$$

The "frustration"

$$
f_{i}=(2 \pi)^{-1} \epsilon^{(\mu \nu)} \nabla_{\nu} A_{i, i+\mu}
$$

describes the magnetic flux through the plaquette $i$, measured in units of the flux quantum $\Phi_{0}=\pi c / 2 e$. Note that due to the fact that the charges and vorticities are integer-valued, only the fractional parts of both offset charges $q_{\mathrm{x} i}$ and frustrations $f_{i}$ matters.

The presence of offset charges $Q_{\mathrm{x} i}=2 e q_{\mathrm{x} i}$, breaking the particle-hole symmetry, has profound consequence on the vortex motion. Offset charges at positions $\vec{r}_{i}$ are responsible for a gauge potential

$\vec{A}(\vec{r})=\sum_{i} q_{\mathrm{x} i} \vec{\nabla} \Theta\left(\vec{r}-\vec{r}_{i}\right)$,

which acts on the vortices in the same way as an ordinary vector potential acts on charges. Associated with this 'vector potential' $\vec{A}(\vec{r})$ is a 'magnetic field' and a 'Lorentz force' acting on moving vortices. We call this force a Magnus force. A vortex with vorticity $v_{n}$ and velocity $\dot{\vec{R}}$ feels the Magnus force [36]

$\vec{F}_{\mathrm{M}}=v_{n} q_{\mathrm{x}} \hat{z} \times \dot{\vec{R}}$.

Here we assumed for simplicity a homogeneous gate charge. As a result of the combined effect of the Magnus force (52) and the Lorentz force, which is the force on the vortex due to an external feeding current, the vortices will move at a certain angle with respect to the current. This angle is called Hall angle. Its measurement yields information on the different forces in the system.

Real samples are usually characterized by random offset charges. As a result the Magnus force averages to approximately zero. This effect is probably responsible for the small size of the observed Hall angle in Josephson junction arrays. The forces on vortices in Josephson junction arrays have been discussed recently in the literature, in part in a controversial way (see Ref. [3741]). Here we want to stress that only the offset 
charges, which are responsible for a local deviations from charge neutrality in the array, lead to the Magnus force.

\section{Conclusions}

We have considered two-dimensional Josephson junction arrays. In the classical limit, $E_{\mathbf{J}} \gg E_{\mathrm{C}}$, the vortices are the relevant excitations. In the opposite quantum limit $E_{\mathrm{C}} \gg E_{\mathbf{J}}$ the charges of the islands are well-defined degrees of freedom. Both can be described simultaneously by a coupled-Coulomb-gas action, which is dual under the interchange of charges and vortices. In each of the two limiting cases $\left(E_{\mathbf{J}} \ll / \gg E_{\mathrm{C}}\right)$ the system reduces to a one-component Coulomb gas, where either the charges or the vortices can be considered as (logarithmically) interacting massive quantum particles. Furthermore, the external magnetic field plays the same role for charges, as the external ("offset") charges for vortices.

The situation is even more interesting in a system of two parallel, capacitively coupled Josephson junction arrays. It is described by a coupledCoulomb-gas action for charges and vortices in both arrays. If one array is in the semi-classical (vortex) regime, while the other is in the quantum (charge) regime we can integrate out the strongly fluctuating variables. In this case we arrive at an effective action, describing dual charges in one array and vortices in the other, which both are now dynamic degrees of freedom, in contrast to the one-layer problem. Furthermore, vortices and charges interact via gauge field of strength controlled by the interlayer capacitance. This peculiar interaction between charges and vortices resembles the composite fermion scenario of the fractional quantum Hall effect, which may become a subject of future research.

\section{Acknowledgments}

The work was supported by the Deutsche Forschungsgemeinschaft within the research program of SFB 195 and by the Swiss National Science Foundation (Y. M. B.).

\section{Appendix}

In this Appendix we provide the steps leading from Eqs. (9), (10) to Eqs. (11), (12). Starting from the partition function (9), we first introduce the vortex degrees of freedom. This can be done by means of the Villain transformation [42] (see also [3]); the time-dependent quantum problem requires some additional steps $[14,27]$. We introduce the lattice with spacing $\epsilon$ in time direction; this spacing is of order of inverse Josephson frequency: $\epsilon \sim\left(8 E_{\mathbf{J}} E_{\mathrm{C}}\right)^{1 / 2}$. In the Villain approximation one replaces

$$
\begin{gathered}
\exp \left\{-\epsilon E_{\mathbf{J}} \sum_{\langle i j\rangle, \tau}\left[1-\cos \left(\phi_{i, \tau}-\phi_{j, \tau}\right)\right]\right\} \\
\rightarrow \sum_{\left\{\mathbf{m}_{i \tau}\right\}} \exp \left\{-\frac{\epsilon E_{\mathbf{J}} F\left(\epsilon E_{\mathbf{J}}\right)}{2} \sum_{i, \tau}\left|\nabla \phi_{i \tau}-2 \pi \mathbf{m}_{i \tau}\right|^{2}\right\} .
\end{gathered}
$$

Here we have introduced a two-dimensional vector field $\mathbf{m}_{\mathbf{i} \tau}$, defined on dual lattice (alternatively, it can be considered as a scalar field defined on bonds). The function

$$
F(x)=\frac{1}{2 x \ln \left(J_{0}(x) / J_{1}(x)\right)} \rightarrow \frac{1}{2 x \ln (4 / x)}, \quad x \ll 1,
$$

is introduced to "correct" the Villain transformation for small $E_{\mathbf{J}}$ (see e.g. Ref. [43]). As we see, its entire effect is to renormalize (increase) the Josephson coupling $E_{\mathbf{J}} \rightarrow E_{\mathbf{J}} F\left(\epsilon E_{\mathbf{J}}\right)$, but it does not affect the physics. In the following we will use only the renormalized constant.

The rhs. of Eq. (A1) can be rewritten as

$$
\sum_{\left\{\mathbf{J}_{i \tau}\right\}} \exp \left\{-\frac{1}{2 \epsilon E \mathbf{J}} \sum_{i, \tau}\left|\mathbf{J}_{i \tau}\right|^{2}-i \mathbf{J}_{\mathbf{i} \tau} \nabla \phi_{i \tau}\right\}
$$

Now the Gaussian integration over the phases can be easily performed, yielding

$$
\begin{aligned}
Z=\sum_{q_{i \tau}} \sum_{\mathbf{J}_{i \tau}} & \exp \left\{-2 e^{2} \epsilon \sum_{i, j, \tau} q_{i \tau} C_{i j}^{-1} q_{j \tau}\right. \\
& \left.-\frac{1}{2 \epsilon E_{\mathbf{J}}} \sum_{i, \tau}\left|\mathbf{J}_{i \tau}\right|^{2}\right\}
\end{aligned}
$$

and the summation is constrained by the continuity equation,

$$
\nabla \mathbf{J}_{i \tau}-\dot{q}_{i \tau}=0
$$


The time derivative stands for a discrete derivative $\dot{f}(\tau)=\epsilon_{\mu}^{-1}\left[f\left(\tau+\epsilon_{\mu}\right)-f(\tau)\right]$. The constraint is satisfied by the parameterization [44]

$$
J_{i \tau}^{(\mu)}=n^{(\mu)}(\mathbf{n} \nabla)^{-1} \dot{q}_{i \tau}+\epsilon^{(\mu \nu)} \nabla_{\nu} A_{i \tau} .
$$

Here the operator $(\mathbf{n} \nabla)^{-1}$ is the line integral on the lattice (in Fourier space it has the form $\left.i\left(k_{x}+k_{y}\right)^{-1}\right), \epsilon^{(\mu \nu)}$ is the antisymmetric tensor, while $A_{i \tau}$ is an unconstrained integer-valued scalar field.

With the use of the Poisson resummation (which requires introducing a new integer scalar field $v_{i \tau}$ ) the partition function can be rewritten as

$$
Z=\sum_{q_{i \tau}, v_{i \tau}} \exp -S\{q, v\}
$$

The effective action for the integer charges $q_{i}$ and vorticities $v_{i}$ is

$$
\begin{aligned}
& S\{q, v\}=\left\{2 e^{2} \epsilon \sum_{i j \tau} q_{i \tau} C_{i j}^{-1} q_{j \tau}\right. \\
& -\frac{1}{2 \epsilon E_{\mathbf{J}}} \sum_{i \tau}\left[n^{(\mu)}(\mathbf{n} \nabla)^{-1} \dot{q}_{i \tau}\right]^{2} \\
& -\frac{\epsilon E_{\mathbf{J}}}{4 \pi} \sum_{i j \tau}\left[2 \pi v_{i \tau}-\frac{i}{\epsilon E_{\mathbf{J}}} \epsilon^{(\mu \nu)} \nabla_{\nu} n^{(\mu)}(\mathbf{n} \nabla)^{-1} \dot{q}_{i \tau}\right] G_{i j} \\
& \left.\times\left[2 \pi v_{i \tau}-\frac{i}{\epsilon E_{\mathbf{J}}} \epsilon^{(\mu \nu)} \nabla_{\nu} n^{(\mu)}(\mathbf{n} \nabla)^{-1} \dot{q}_{j \tau}\right]\right\}
\end{aligned}
$$

The kernel $G_{i j}$ is the lattice Green's function, i.e. the Fourier transform of $k^{-2}$. Finally, after some algebra [27] we arrive to the effective action (12), rewritten in the continuous notations.

\section{REFERENCES}

1. H. A. Kramers and G. H. Wannier, Phys. Rev. 60, 252 (1941).

2. R. Savit, Rev. Mod. Phys. 52, 453 (1980).

3. J. V. José, L. P. Kadanoff, S. Kirkpatrick, and D. R. Nelson, Phys. Rev. B 16, 1217 (1977).

4. L. P. Kadanoff, J. Phys. A 11, 1399 (1978).

5. B. Nienhuis, in: Phase transitions and critical phenomena, Vol. 11, ed. by C. Domb and J. L. Lebowitz (Academic Press, London, 1987), p.1.
6. see e.g. Proceedings of the NATO ARW Coherence in Superconducting Networks, J.E. Mooij and G. Schön eds., Physica B152, (1988); J.E.Mooij and G. Schön, in: Single Charge Tunneling, H. Grabert and M. H. Devoret eds. (Plenum, N. Y., 1992).

7. J. M. Kosterlitz and D. J. Thouless, J. Phys. C 6, 1181 (1973).

8. B. I. Halperin and D. R. Nelson, J. Low. Temp. Phys. 36, 599 (1979).

9. V. L. Berezinskii, Zh. Eksp. Theor. Fiz. 59, 907 (1970) [Sov. Phys. - JETP 32, 493 (1971)].

10. E. H. Hauge and P. C. Hemmer, Phys. Norvegica 5, 209 (1971).

11. J. M. Kosterlitz, J. Phys. C 7, 1046 (1974).

12. P. Minnhagen, Rev. Mod. Phys. 59, 1001 (1987).

13. G. Schön and A. D. Zaikin, Phys. Reports 198, 237 (1990).

14. R. Fazio and G. Schön, Phys. Rev. B 43, 5307 (1991).

15. F. Guinea and G. Schön, J. Low. Temp. Phys. 69, 219 (1987); G. Schön and A. D. Zaikin, Physica B 152, 203 (1988); S. M. Apenko, Phys. Lett. A 142, 277 (1989).

16. B. Abeles, Phys. Rev. B 15, 2828 (1977).

17. K. B. Efetov, Zh. Eksp. Theor. Fiz. 78, 2017 (1980) [Sov. Phys. - JETP 51, 1015 (1980)].

18. E. Šimánek, Phys. Rev. Lett. 45, 1442 (1980).

19. S. Doniach, Phys. Rev. B 24, 5063 (1981).

20. Yu. E. Lozovik and S. G. Akopov, J. Phys. C 14, L31 (1981).

21. L. J. Jacobs, J. V. José and M. A. Novotny, Phys. Rev. Lett. 53, 2177 (1984).

22. R. S. Fishman and D. Stroud, Phys. Rev. B 38, 290 (1988).

23. J. V. José, Phys. Rev. B 29, 2836 (1984); see also J. V. José and C. Rojas, Physica B 203, 481 (1994).

24. M. .C. Diamantini, P. Sodano and C. A. Trugenberger Phys. Rev. Lett. 75, 3517 (1995).

25. M. P. A. Fisher, G. Grinstein, and S. M. Girvin, Phys. Rev. Lett. 64, 587 (1990).

26. J. E. Mooij, B. J. van Wees, L. J. Geerligs, M. Peters, R. Fazio, and G. Schön, Phys. Rev. Lett. 65, 645 (1990).

27. R. Fazio, U. Geigenmüller, and G. Schön, 
in Quantum Fluctuations in Mesoscopic and Macroscopic Systems, H. A. Cerdeira, et al. eds. (World Scientific, 1991), p. 214.

28. See the appendix of: D. R. Nelson and B. I. Halperin, Phys. Rev. B 21, 5312 (1980).

29. It is easy to show that the vortices with $\left|v_{n}\right|>$ 1 are unstable and decay to separate vortices with $v_{n}= \pm 1$.

30. E. Šimánek, Sol. State. Commun. 48, 1023 (1983).

31. U. Eckern and A. Schmid, Phys. Rev. B 39, 6441 (1989).

32. In 1D Josephson chains, in the presence of extra inductive terms charge solitons with a larger mass may appear as topological excitations. See Z. Hermon, E. Ben-Jacob, and G. Schön, Phys. Rev. B 54, 1234 (1996).

33. This situation is quite different from multilayered systems with Josephson coupling between layers, that have been discussed in the literature: see e.g. S. R. Shenoy, Phys. Rev. B 42, 8595 (1990); N. K. Kultanov and Yu. E. Lozovik, Sol. St. Commun. 88, 645 (1993). The analog of the BKT transition in these systems is the disruption of vortex rings. In the limit of weak Josephson coupling this system is reduced to the $2 \mathrm{D}$ XY-model, while in the opposite case of strong coupling it is essentially the 3D XY-model.

34. Ya. M. Blanter and G. Schön, Phys. Rev. B 53, 14534 (1996).

35. C. Rojas, J. V. José, and A. M. Tikofsky, Bull. Am. Phys. Soc. 40, p.68, B11-7 (1995); J. V. José, private communication.

36. R. Fazio, A. van Otterlo, G. Schön, H. S. J. van der Zant, and J. E. Mooij, Helv. Physica Acta 65, 228 (1992).

37. F. Gaitan and S. R. Shenoy, Phys. Rev. Lett. 76, 4404 (1996).

38. X.-M. Zhu, Y. Tan, and P. Ao, condmat/9507126.

39. Yu. G. Makhlin and G. E. Volovik, Pis'ma Zh. Eksp. Teor. Fiz. 62, 923 (1985) [JETP Lett. 62, $941(1995)]$.

40. E. B. Sonin, cond-mat/9606099; Phys. Rev. $\mathrm{B}$, to be published.

41. A. van Otterlo, G. Schön, and G. Blatter, Comment submitted to Phys. Rev. Lett.
42. J. Villain, J.Physique 36, 581 (1975).

43. A. van Otterlo, K.-H. Wagenblast, R. Baltin, C. Bruder, R. Fazio, and G. Schön, Phys. Rev. B 52, 16176 (1995).

44. S. Elitzur, R. Pearson, and J. Shigemitzu, Phys. Rev. D 19, 3638 (1979). 